\title{
Estimation of Applied Forces on Railway Vehicle Wheelsets from Measured Vehicle Responses
}

\author{
Majid Mehrpouya a and Hamid Ahmadian \\ School of Mechanical Engineering, \\ Iran University of Science and Technology, Tehran, 16846-13114, Iran. \\ ${ }^{a}$ Corresponding Author,Email: majid_mehrpouya@mecheng.iust.ac.ir
}

\begin{abstract}
:
In this paper, a finite element (FE) model of a railway freight vehicle is proposed to be used in the identification of applied forces to the wheelsets. The proposed model is comprised of two, two-axle bogies of type H665 and a freight wagon. The model is updated in two stages. First, the bogie is updated by using modal data extracted from measurements on an actual bogie separated from wagon. Secondly, the whole structure of the vehicle is updated by modal data obtained from a test conducted on the vehicle in the laboratory. Since the proposed model is relatively large, it is reduced to some desirable points through the superelement analysis method, in order to reduce the cost of computation. The final reduced model is then used to identify the applied forces resulted from irregularities of track.
\end{abstract}

\section{KEYWORDS:}

Railway Vehicle, Superelement Analysis, Force Identification

\section{CITATION:}

M. Mehrpouya and H. Ahmadian. 2009. Estimation of Applied Forces on Railway Vehicle Wheelsets from Measured Vehicle Responses, Int. J. Vehicle Structures \& Systems, 1(4), 104-110.

\section{Introduction}

Nowadays, a great proportion of the railways industry budget is allocated to maintenance, keeping facilities such as track and rolling stock in good condition. The importance of this issue is highlighted when phenomena such as derailment that results from poor track condition causes many people to be wounded or die annually all around the world. Many companies use track inspection vehicle to ensure railroad safety. These vehicles are equipped with special mechanisms and sensors to measure the track geometry directly. However, these systems impose high expenses on companies because of both their high costs of purchase and maintenance. Therefore, alternatives of these traditional ways are now of great concern. Bleakley [1] used signal data obtained during the normal operation of vehicle on a track to define unsafe territories of track by comparing these data with the available standards. This method is generally used for determination of hazardous areas and no information is obtained about the applied forces.

Xia et al. [2] have provided an inverse wagon model to estimate wheel-rail contact forces using only measurements of wagon body responses as inputs. In their work, both wagon body and bogies are modelled with rigid beams and the partial modal matrix (PMM) method is used to predict the input loads. Kawasaki and Youcef-Toumi [3] have provided a method to estimate the rail irregularities by measuring accelerations of a passenger car using an auto regressive model with extra inputs (ARX). They used data from a track with known irregularities and the accelerations of the car recorded during movement on that track to identify the parameters of their model. Uhl [4] used a dynamic programming optimization method to minimize the objective function, which is the least square error between the simulated and measured system responses, in order to identify the excitation forces applied to the wheels of a rail vehicle.

Since direct measurement of applied forces on many structures either requires expensive instrumentation or inapplicable because of inaccessibility of applied locations, force identification methods are of great concern in the literature. Some of these methods are based on time domain approaches. Force determination in time domain uses an expansion method to estimate unmeasured responses of structure from the measured ones. By having all the responses, it is possible to solve the governing equation of system backward to reconstruct the applied forces. Genaro [5] used this method for identification of applied forces to a case study system of 11 degrees of freedom (DOF).

Some other methods in frequency domain are generally based on the pre-multiplication of frequency spectrum of measured responses of the system by inverse Frequency Response Function (FRF) matrix [6]. Leclere et al. [7] used this method to determine the power spectrum of bearing loads of a diesel engine from the power spectral density of measured responses. Okubo [8] also used this method for determination of excitation forces of an automobile engine. This method is also used by Uhl [9] for the identification of in-flight loading forces from measured responses.

In this work, an updated Finite Element (FE) model of railway vehicle to be used in force identification 
procedure is proposed. The model is then reduced through a dynamic reduction procedure to decrease the computation costs. Responses of vehicle in some specific points on the car body are recorded and then transformed in frequency domain. Regarding the methods in frequency domain, the applied forces to wheelsets are reconstructed by pre-multiplication of frequency spectrum of measured responses by inverse FRF matrix of the system.

\section{Finite Element Modelling}

The bogie used in this work is a two-axel bogie of type H665. This type of bogie is frequently used in freight vehicles. The majority parts of the bogie are comprised of plates of different thicknesses which are welded to each other to form the main structure. To validate the accuracy of proposed FE model, it is improved by natural frequencies obtained from measurements on an actual bogie using FE model updating procedure. The final results obtained from the improved analytical model conform to the measured ones [10].

The skeleton of the car body consists 8 cross sectional beams that are used to stiffen the structure. Between these beams, walls are located which are made from steel panels of $6 \mathrm{~mm}$ thick. All of the stiffener beams are modelled by beam elements with constant cross sections and the walls are modelled by shell elements with properties of the steel. Between the first and second cross sectional spans there is no wall, but doors of the car body are located. These doors are modelled as lumped masses with equivalent moment of inertia with their masses distributed on the hinges. Connection of each bogie to the body is provided by 3 springs. Two linear springs are used to model the suspensions in vertical and lateral directions which resist against the pitch and yaw modes respectively. One rotational spring is used to model the suspension about longitudinal axis which resists against the roll mode of body. The stiffness values of these springs are obtained through the FE model updating procedure of the entire structure.

In order to extract the modal properties of vehicle, the car body is excited by a lever that is located between one of the bogies and car body. The car body is excited with a hammer that directly targeted its body. In both of these tests, 15 uni-axial accelerometers are used to record the accelerations of the structure on its walls and floor [11]. Accelerometers are arranged in an order that detection of rigid body modes is possible as well as detection of elastic modes of the car body in lateral and vertical directions. Fig. 1 shows the locations and directions of accelerometers.

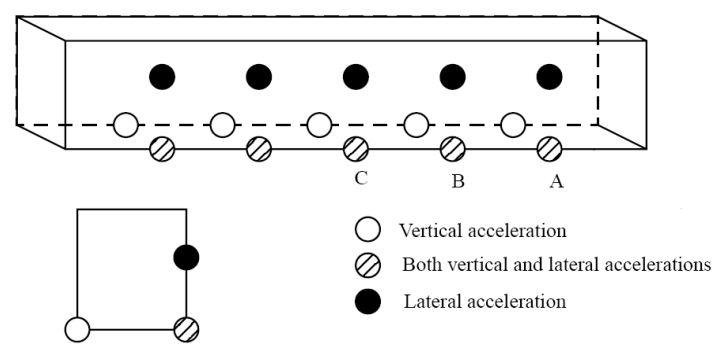

Fig. 1: Location of uni-axial accelerometers
Frequency Domain Decomposition (FDD) method is used for identification of the natural frequencies and mode shapes of the structure. Linearized sensitivity method is used for the FE model updating procedure. The basis of this method is the assumption that the difference between the measured and predicted modal properties (natural frequencies), can be described in terms of relevant modal sensitivities (rates of change of natural frequencies $\left(\delta \lambda_{i} / \delta p_{i}\right)$ with respect to changes in individual variable terms $p_{i}$ ) and small adjustments to the selected design variables in the model $\left(\Delta p_{i}\right)$ [12]. This can be expressed as:

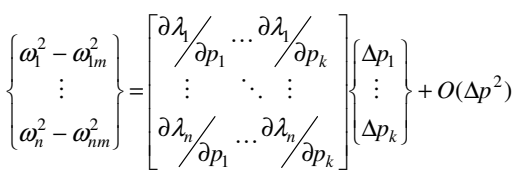

Where

$\frac{\partial \lambda_{n}}{\partial p_{k}}=\frac{\phi_{n}^{T}\left(\partial K / \partial p_{k}-\lambda_{n} \partial M / \partial p_{k}\right) \phi_{n}}{\phi_{n}^{T} M \phi_{n}}$

where $M$ and $K$ are respectively the mass and stiffness matrices and $\phi_{n}$ is the eigenvector of the system.

FE model updating procedure is stopped after 5 iterations. After this, the stiffness values of the springs between bogies and body are obtained as $k_{-} y=1 . E 10 \mathrm{~N} / \mathrm{m}$ and $k_{-} z=6.44 E 5 \mathrm{~N} / \mathrm{m}$ respectively for the linear spring in vertical and lateral directions, and $k r_{-} x=2.17 E 6 \mathrm{Nm}$ for one rotational spring about longitudinal axis respectively. Table 1 gives the natural frequencies of the structure before and after FE model updating procedure along with the measured ones. The comparison of measure frequencies with the predicted ones from FE model shows a good agreement. The predicted (FE model updated) mode shapes of whole vehicle car body at these frequencies are shown in Figs. 2-6 respectively. Also, the first 5 mode shapes (undeformed structure in gray mesh) at the middle section of the bogie is shown respectively in Figs. 7 to 11.

Table 1: Natural frequencies of structure

\begin{tabular}{cccc}
\hline Mode & $\begin{array}{c}\text { Before } \\
\text { updating }(\mathrm{Hz})\end{array}$ & $\begin{array}{c}\text { After } \\
\text { updating }(\mathrm{Hz})\end{array}$ & $\begin{array}{c}\text { Measured } \\
(\mathrm{Hz})\end{array}$ \\
\hline Roll & 1.69 & 2.48 & 2.66 \\
$1^{\text {st }}$ Elastic & 4.09 & 4.23 & 4.66 \\
Pitch & 4.87 & 8.04 & 8.33 \\
$2^{\text {nd }}$ Elastic & 13.58 & 14.23 & 14.00 \\
$3^{\text {rd }}$ Elastic & 24.70 & 24.87 & 24.66 \\
\hline
\end{tabular}

\section{Superelement Analysis}

In FE analysis, the demand for computer resources will always exceed existing capabilities. By using superelements, one can not only analyze large models, but also can become more efficient in performing the analysis, thereby allowing more design cycles or iterations in the analysis. The principle used in superelement analysis is substructuring where the model is divided into a series of components (superelements), each of which is processed independently, resulting in a set of reduced matrices that describe the behaviour of the superelement as seen by the rest of the structure. 


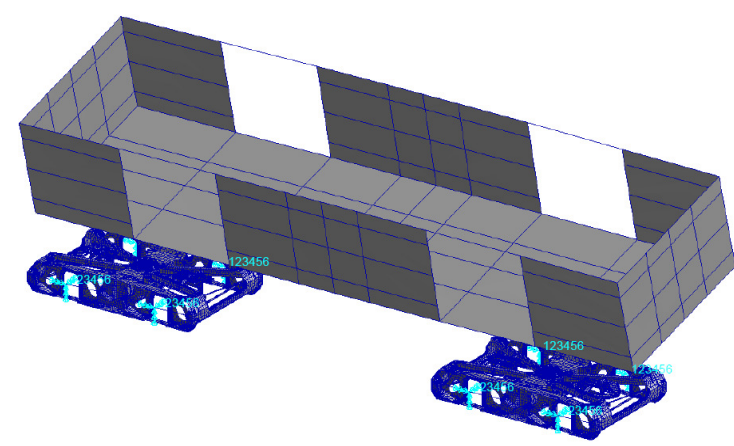

Fig. 2: Roll mode shape from FE model

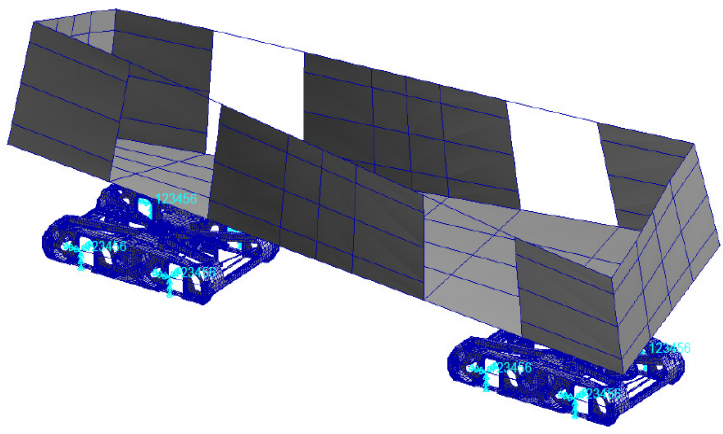

Fig. 3: First elastic mode shape from FE model - Whole vehicle

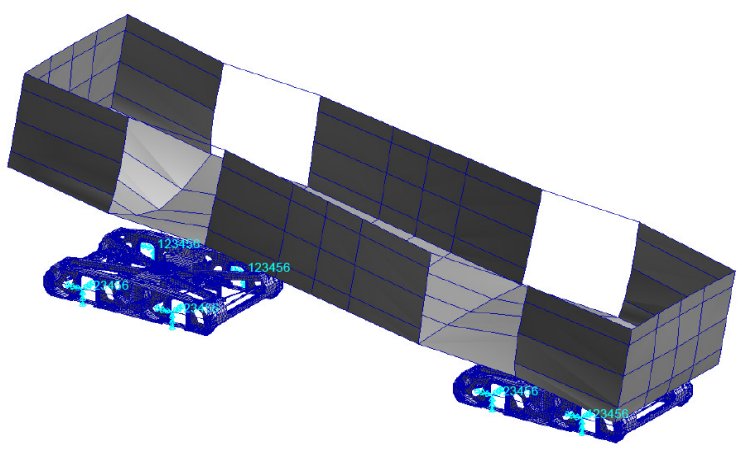

Fig. 4: Pitch mode shape from FE model - Whole vehicle

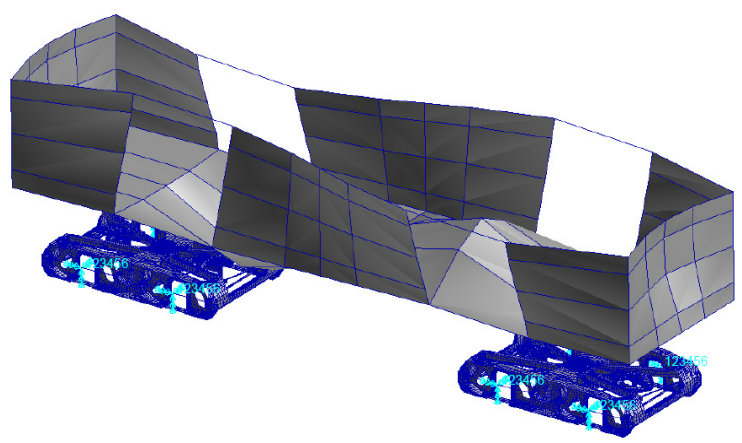

Fig. 5: Second elastic mode shape from FE model - Whole vehicle

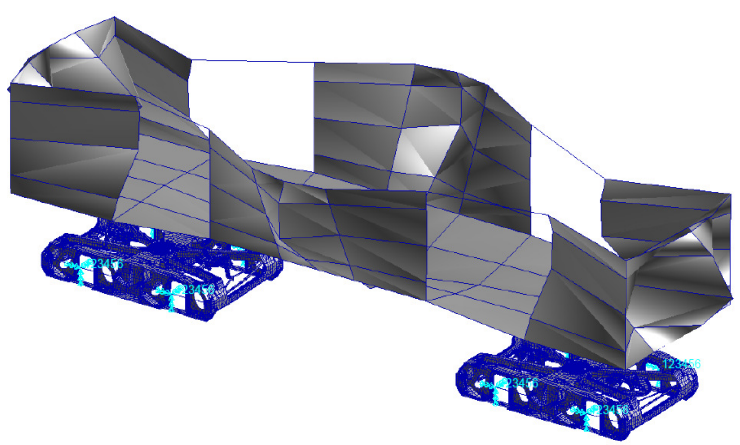

Fig. 6: Third elastic mode shape from FE model - Whole vehicle

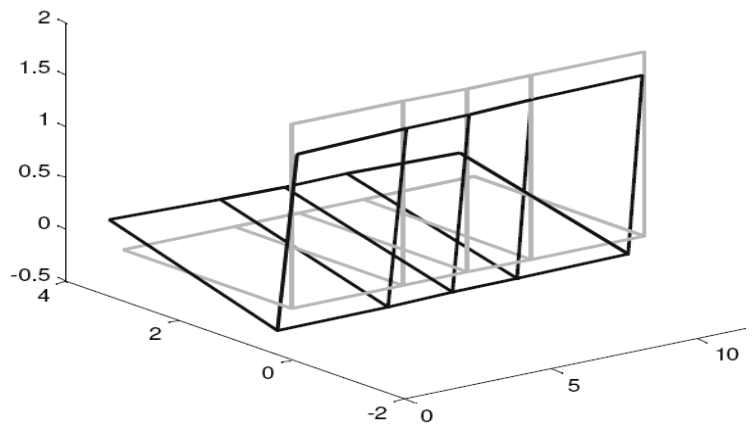

Fig. 7: Roll mode shape from FE model - Car body section

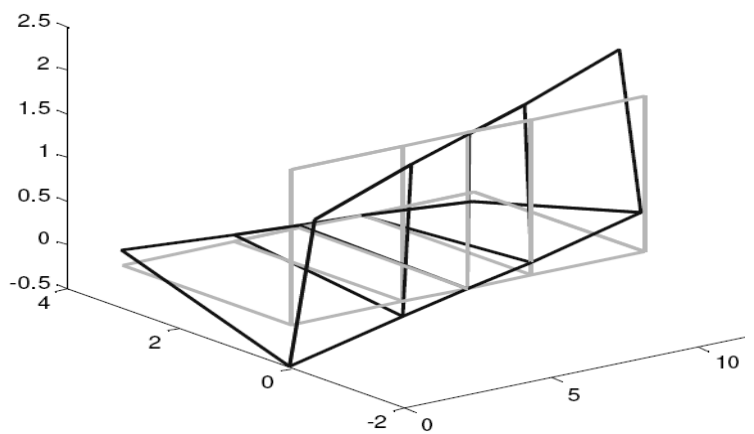

Fig. 8: First elastic mode shape from FE model - Car body section

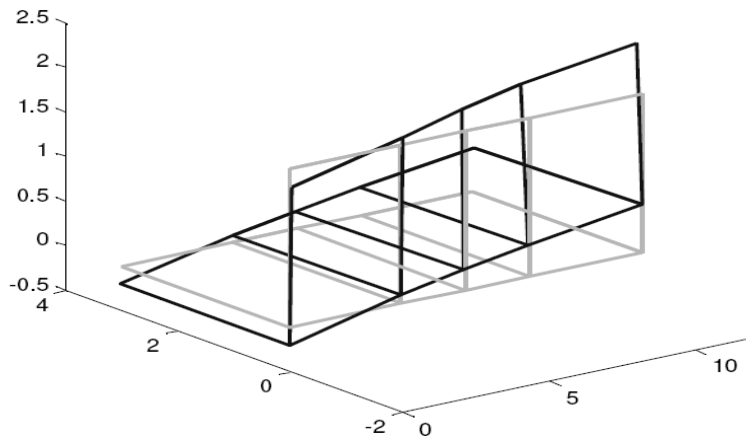

Fig. 9: Pitch mode shape from FE model - Car body section

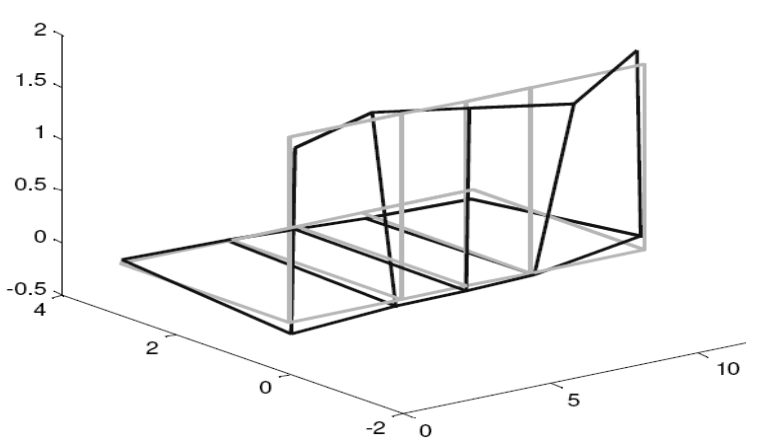

Fig. 10: $2^{\text {nd }}$ Elastic mode shape from FE model - Car body section

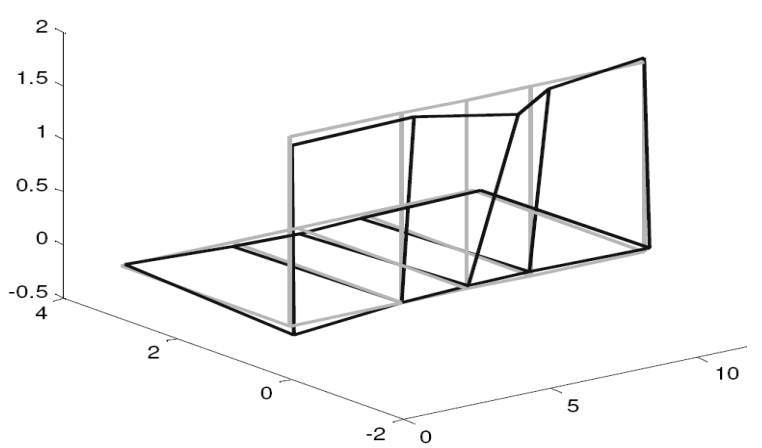

Fig. 11: $3^{\text {rd }}$ Elastic mode shape from FE model - Car body section 
The reduced matrices for the individual superelements are combined to form an assembly (or residual) solution. The results of the assembly solution are then used to perform data recovery (calculation of displacements, stresses, etc.) for the superelements [13]. In dynamic reduction the assumption is that the solution for each superelement can be represented by using superposition of a series of shape functions:

$$
U_{o}=G_{o t} \cdot U_{t}+G_{o q} \cdot U_{q}
$$

where $U_{o}$ is the motion of the interior points of the current superelement. $G_{o t}$ and $G_{o q}$ are the static and dynamic transformation matrices. $U_{t}$ and $U_{q}$ are the solutions for the motion of the physical and generalized exterior DOFs respectively. Interior points are the ones which are not desirable and are omitted after the reduction. On the other hand, exterior points are the ones on which the reduced matrices are based and the entire structure is reduced to these points. In the case of fixedboundary dynamic reduction (better known as CraigBampton Component Mode Synthesis), $G_{o t}$ is the static motion of the superelement resulting from moving one boundary (exterior) DOF by 1.0 unit while holding the other boundary DOFs fixed and $G_{o q}$ is the eigenvector of the system obtained from the original system after its boundary nodes are fixed.

In this work, the entire structure is reduced to 15 points on the body where accelerometers are attached and 4 points on each bogie where the suspension springs are attached to the wheelsets. The reduced model is then used in force identification procedure instead of the entire FE model to reduce the cost of computation. The response of structure is detected by those 15 points on the accelerometer positions. The forces from wheelsets, which we are interested in defining, are applied at those 8 points on the bogies. Since the wheelsets are connected to bogies through suspension springs, having the knowledge of applied forces to the points where springs attach to bogie, can lead us to the applied forces on the wheelsets that are directly proportional to track irregularities.

A comparison between the natural frequencies of structure obtained from normal mode analysis of the complete model and those obtained from the reduced model is given in Table 2. Moreover, the mode shapes corresponding to these 6 modes are in good agreement with each other, i.e., the norm of difference between mode shape of the complete model and the reduced model in each mode is close to zero. This shows that reduced model can be reliably used instead of full model.

Table 2: Natural frequencies of structure

\begin{tabular}{ccc}
\hline Mode No. & $\begin{array}{c}\text { Complete model } \\
(\mathrm{Hz})\end{array}$ & $\begin{array}{c}\text { Reduced model } \\
(\mathrm{Hz})\end{array}$ \\
\hline 1 & 2.010549 & 2.010541 \\
2 & 2.479246 & 2.479240 \\
3 & 4.229781 & 4.229772 \\
4 & 4.585932 & 4.585439 \\
5 & 8.039200 & 8.039191 \\
6 & 10.20173 & 10.20162 \\
\hline
\end{tabular}

\section{Force Identification}

For a multi-degree of freedom system with $N$ degrees of freedom, the governing equations of motions can be written as [12]:

$$
[M]\{\ddot{x}\}+[C]\{\dot{x}\}+[K][x\}=\{f\}
$$

where $[M],[C]$ and $[K]$ are the mass, damping and stiffness matrices respectively. $\{f\}$ and $\{x\}$ are timedependent vectors of force and displacement. If the structure is excited by a set of forces at the same frequency of $\omega$ but with individual amplitudes and phases, $\{f(t)\}=\{F\} e^{i \omega t}$, then the solution of problem will be in the form of $\{x(t)\}=\{X\} e^{i \omega t}$. Therefore, the equation of motion will change to the form of:

$$
\left([K]-\omega^{2}[M]+i \omega[C]\right)\{X\}=\{F\}
$$

Rearranging Eqn. 5 results in:

$$
\{X\}=\left([K]-\omega^{2}[M]+i \omega[C]\right)^{-1}\{F\}
$$

This may be written as:

$$
\{X\}=[H(\omega)]\{F\}
$$

where $[H(\omega)]$ is $N \times N$ receptance FRF of the system and can be obtained from Eqns. 6 and 7 using:

$$
\left([K]-\omega^{2}[M]+i \omega[C]\right)=[H(\omega)]^{-1}
$$

Eqn. 8 is premultiplied by $[\Phi]^{T}$ on both sides and also postmultiplied $[\Phi]$ on both sides and lead to:

$$
[H(\omega)]=[\Phi]\left[\omega_{r}^{2}-\omega^{2}+2 i \xi_{r} \omega_{r} \omega\right]^{-1}[\Phi]^{T}
$$

Eqn. 9 permits us to compute any individual FRF parameter, $H_{j k}(\omega)$, using the following formula:

$$
H_{j k}(\omega)=\sum_{r=1}^{N} \frac{\phi_{j r} \phi_{k r}}{\omega_{r}^{2}-\omega^{2}+2 i \xi_{r} \omega_{r} \omega}
$$

According to Eqn. 7, the multiplication of the FRF matrix with the vector of excitation forces yields the response of these forces on a structure. It should be noted that the FRF matrix, the force, and the response vectors are all functions of frequency $\omega$. By multiplying both sides of Eqn. 7 by $\left([\mathrm{H}(\omega)]^{-1}\right)$, the foundation of the so-called FDD force estimation method will be formed. The new equation leads into the determination of excitation forces by using the FRF matrix and vibration response levels as:

$$
\{F(\omega)\}=[H(\omega)]^{-1}\{X(\omega)\}
$$

The most challenging part of this procedure is the construction of the FRF matrix and taking its inverse with an acceptable accuracy. Since FRF matrix represents the dynamic properties of a structure, it is crucial to get FRFs measured or calculated with high accuracy. It is noteworthy to say that the number of responses are about to be measured $(m)$ and the number of forces are about to be determined $(n)$ are two critical parameters during the solution of this inverse problem. To avoid ill-conditioning of FRF matrix, it is recommended that the number of measured response shall be greater than the number of applied forces, i.e., $m>n$. In this work we have 8 points where suspension 
springs attach to the wheelsets and forces are applied to the structure and 15 points on the vehicle body where the responses are detected. Since the FRF matrix is not square in this case, we use a pseudo-inverse of FRF matrix instead of its inverse, leading Eqn. 11 to:

$$
\{F(\omega)\}=[H(\omega)]^{+}\{X(\omega)\}
$$

\section{Results and Discussions}

In order to investigate the accuracy of the proposed method, a vertical force with frequency content of 10, 5, and $2 \mathrm{~Hz}$ is applied to the structure using:

$$
F=500 \cos (20 \pi t)+200 \cos (10 \pi t)+800 \cos (4 \pi t)
$$

and the recorded responses of the body at the points shown in Fig. 1 are used in the reconstruction of applied forces. A sample response of one of the points in the time and frequency domain is shown in Fig. 12 and 13 respectively. Using the responses of other points, the constructed force is obtained in frequency domain. Reconstructed force in comparison with applied force is shown in Fig. 14.

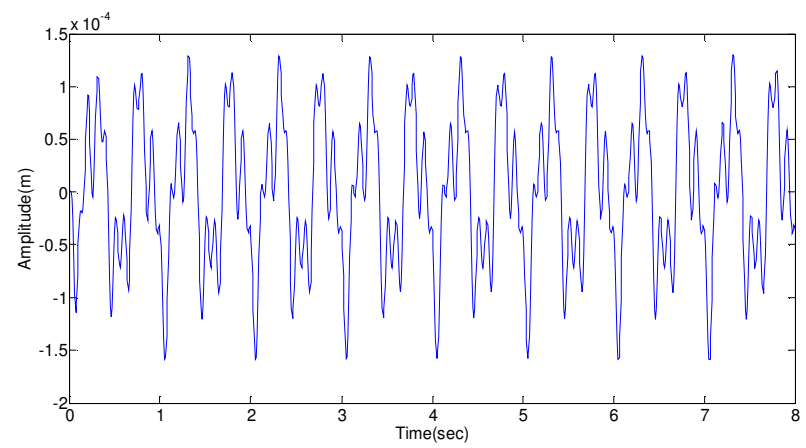

Fig. 12: Same response in time domain

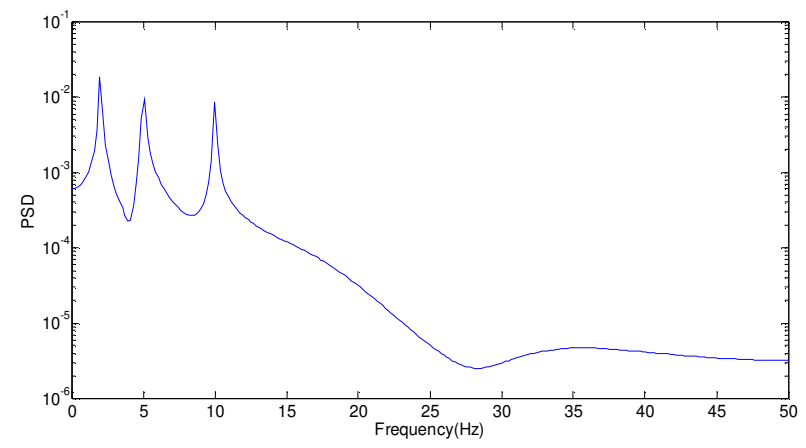

Fig. 13: Sample response in frequency domain

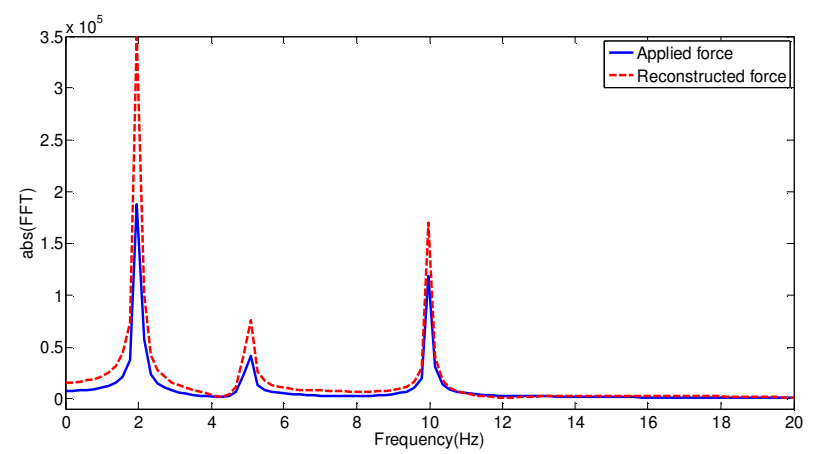

Fig. 14: Comparison of reconstructed and actual force
As a second case, two forces - a vertical force $\left(F_{y}\right)$ with frequency content of 2 and $4 \mathrm{~Hz}$ and a lateral force $\left(F_{z}\right)$ with frequency content of $10 \mathrm{~Hz}$ are applied simultaneously to the structure on different points using:

$$
\begin{aligned}
& F_{y}=500 \cos (4 \pi t)+200 \cos (8 \pi t) \\
& F_{z}=300 \cos (20 \pi t)
\end{aligned}
$$

By applying the same procedure, it is possible to reconstruct these forces from measured responses. Fig. 15 shows the comparison between vertical applied force and reconstruction of this force.

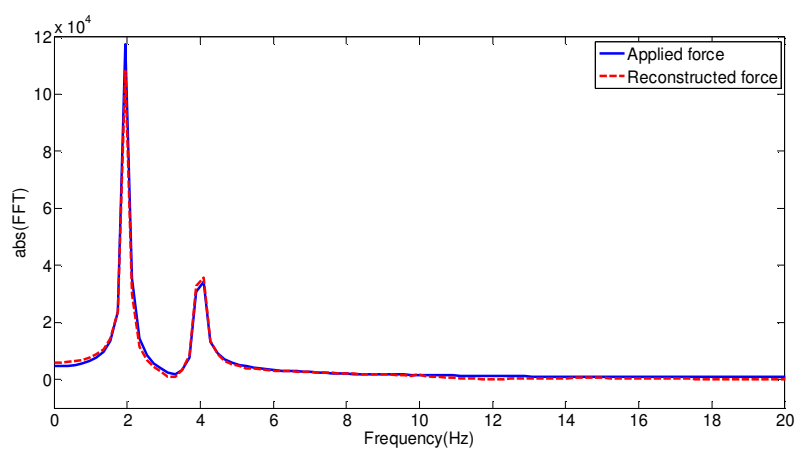

Fig. 15: Comparison of reconstructed and actual force

\section{Conclusions}

In this paper FE model of railway freight vehicle is proposed and updated by its modal properties extracted from the measurements on an actual vehicle. The updated model is then used in the force identification procedure. Instead of using a complete FE model, a reduced model, which is obtained after exerting a dynamic reduction procedure suing superelements on the complete model, is used in the force determination procedure. This force identification procedure is conducted in frequency domain which is based on the premultiplication of recorded responses of structure in frequency domain by the inverse FRF matrix of reduced model. This procedure gives a reliable estimation of the applied forces to structure.

It is shown that the forces with low frequency contents can be estimated reliably by the proposed methods. These low-frequency-content forces, which are caused by track deterioration from its normal geometric conditions, when the forces exceed the normal values, are generally the cause of derailment. Since the frequency contents of the responses are used in this procedure, it is recommended that these responses shall be recorded at high resolution to generate consistent frequency content plots. The proposed FE model updating and identification procedure can be used in actual applications. Responses of a real vehicle in its normal operation can be detected and feedback to the model to give an estimation of actual applied forces on the vehicle during the normal operation. By monitoring these forces, it is viable to spot lengths of track where applied forces exceed the normal values defined for safe operation of vehicle. 


\section{REFERENCES:}

[1] S. Bleakley. 2006. Time Frequency Analysis of Railway Wagon Body Acceleration for a Low-Power Autonomous Device, M.Sc. Thesis, Central Queensland University, Australia.

[2] F. Xia, C. Cole, and P. Wolfs. 2007. An inverse railway wagon model and its applications, Vehicle System Dynamics, 45(6), 583-605.

[3] J. Kawasaki and K. Y. Toumi. 2002. Estimation of rail irregularities, Proc. American Control Conf., Anchorage, USA.

[4] T. Uhl. 2007. The inverse identification problem and its technical application, Arch. Appl. Mech., 77, 325-337.

[5] G. Genaro and G.A. Rade. 1998. Input force identification in the time domain, Proc. $16^{\text {th }}$ Int. Modal Analysis Conf., USA.

[6] E. Parloo, P. Verboven, P. Guillaume, and M. Overmeire. 2003. Force identification by means of in-operation modal models, J. Sound \& Vibration, 262, 161-173.

[7] Q. Leclere, C. Pezerat, B. Laulagnet, and L. Polac. 2005. Indirect measurement of main bearing loads in an operating diesel engine, J. Sound \& Vibration, 286, 341361.

[8] N. Okubo, S. Shimamura, and T. Toi. 2006. Identification of excitation force generated from automobile engine in high frequency range, Proc. Int. Seminar Modal Analysis, 3985-3992, Belgium.

[9] T. Uhl and K. Mendrok. 2006. Reconstruction of loading forces from responses data measured in-flight, Proc. Int. Seminar Modal Analysis, 4029-4040, Belgium.

[10] M. Mehrpouya and H. Ahmadian. 2009. Finite element model updating of railway bogie, $17^{\text {th }}$ Int. Conf. Mechanical Engineering, Iran.

[11] H. Ahmadian. 2005. Extracting modes of a railway vehicle from measured responses, $1^{\text {st }}$ Int. Operational Modal Analysis Conf., Denmark.

[12] D.J. Ewins. 2000. Modal Testing: Theory, Practice and Application, Research Studies Press, Baldock, Hertfordshire, UK.

[13] MSC/NASTRAN Superelement User's Guide. 2001. MacNeal Schwendler Corp., Los Angeles, California.

\section{EDITORIAL NOTES:}

Edited paper from $2^{\text {nd }}$ Int. Conf. on Recent Advances in Railway Engineering, 27-28 September 2009, Tehran, Iran.

GUEST EDITOR: Dr. Davood Younesian, School of Railway Engineering, Iran University of Science and Technology, Farjam St, Tehran 16846-13114, Iran. 